\title{
On the Adjacent Eccentric Distance Sum Index of Graphs
}

\author{
Hui $\mathrm{Qu}^{1}{ }^{*}$, Shujuan $\mathrm{Cao}^{2}$ \\ 1 Department of Mathematics, Shandong Institute of Business and Technology, Yantai, Shandong, China, \\ 2 School of Mathematical Sciences, Nankai University, Tianjin, China \\ * quhui781111@126.com
}

\section{Abstract}

For a given graph $G, \varepsilon(v)$ and $\operatorname{deg}(v)$ denote the eccentricity and the degree of the vertex $v$ in $G$, respectively. The adjacent eccentric distance sum index of a graph $G$ is defined as $\xi^{s v}(G)=\sum_{v \in V(G)} \frac{\varepsilon(v) D(v)}{d e g(v)}$, where $D(v)=\sum_{u \in V(G)} d(u, v)$ is the sum of all distances from the vertex $v$. In this paper we derive some bounds for the adjacent eccentric distance sum index in terms of some graph parameters, such as independence number, covering number, vertex connectivity, chromatic number, diameter and some other graph topological indices.

\section{G openaccess}

Citation: Qu H, Cao S (2015) On the Adjacent Eccentric Distance Sum Index of Graphs. PLoS ONE 10(6): e0129497. doi:10.1371/journal.pone.0129497

Academic Editor: Vince Grolmusz, Mathematical Institute, HUNGARY

Received: February 20, 2015

Accepted: May 8, 2015

Published: June 19, 2015

Copyright: $\odot 2015$ Qu, Cao. This is an open access article distributed under the terms of the Creative Commons Attribution License, which permits unrestricted use, distribution, and reproduction in any medium, provided the original author and source are credited.

Data Availability Statement: We confirm that any researchers interested in repeating our experiments or reproving our proofs are able to do so with the information (dataset) provided in our manuscript.

Funding: This work was supported by the Natural Science Foundation of China (Nos. 11301302, $11101245,61202362)$ and the Natural Science Foundation of Shandong (No. BS2013SF009).

Competing Interests: The authors have declared that no competing interests exist.

\section{Introduction}

In theoretical chemistry and biology, molecular structure descriptors or topological indices are used for modeling information of molecules, including physico-chemical, toxicologic, pharmacologic, biological and other properties of chemical compounds. A topological index is a numerical value based on a simple graph, which is an invariant. Topological indices have been found to be useful in chemistry, bioinformatics and network science. Generally, these indices are divided into three classes. One class is degree-base indices [51], such as (general) Randic index [46, 52], (general) zeroth order Randic index [34, 35], Zagreb index [26], ABC index [50]. One class is distance-based indices [61], such as Wiener index [40, 57, 58, 60], Balaban index [4, 5, 16], the Wiener polarity index [24, 49], Szeged index [1], Harary index [2], Hosoya index $[32,33]$. The third class is spectrum-based indices, such as graph energy $[18,27,31,36$, $37,47,48]$, incidence energy $[9,10]$, matching energy $[12,13,43]$, Randic energy $[8,19]$, HOMO-LUMO index [45], Kirchhoff index [44], graph entropies [11, 14, 15, 20, 21, 42, 65]. Recently, some other indices based on eccentricity have been introduced and investigated by mathematicians, chemists and biologists, which contain eccentric connectivity index [28, 41 , $55]$, eccentric distance sum $[29,38,62]$, augmented and super augmented eccentric connectivity indices $[3,25]$, connective eccentricity index $[30,63,64]$, adjacent eccentric distance sum index $[53,54]$ and so on.

Throughout this paper, graphs considered are simple and connected. Let $G$ be a simple connected graph with vertex set $V(G)$. For a vertex $v \in V(G), \operatorname{deg}(v)$ denotes the degree of $v$. For vertices $u, v \in V(G)$, the distance $d(u, v)$ is defined as the length of the shortest path between $u$ 
and $v$ in $G$ and $D_{G}(v)$ (or $D(v)$ for short) denotes the sum of all distances from $v$. The eccentricity $\varepsilon(v)$ of a vertex $v$ is the maximum distance from $v$ to any other vertex. The radius $r(G)$ of a graph is the minimum eccentricity of any vertex, while the diameter $D(G)$ of a graph is the maximum eccentricity of any vertex in the graph. Let $K_{n}$ and $P_{n}$ be the complete graph and path graph on $n$ vertices, respectively. We write $K_{n}-k e$ for the graph obtained from $K_{n}$ by deleting $k$ independent edges.

In chemistry and biology, people want to find some new molecules with desired physicochemical properties, for example, boiling points, molecular volumes, flavor threshold concentration antiviral activity, energy levels, electronic populations, etc. And these properties can be quantitatively represented by some values of a certain index. The notion of a "topological index" appears first in a paper of the Japanese chemist Hosoya [32], who investigated the surprising relation between the physicochemical properties of a molecule and the number of its independent edge subsets (matchings). A topological index is a map from the set of chemical graphs to the set of real numbers. Therefore a topological index is a numeric quantity that is mathematically derived in a direct and unambiguous manner form the structural graph of a molecule. Since isomorphic graphs possess identical values for any given topological index, these indices are referred to as graph invariants. Topological indices usually reflect both molecular size and shape. The advantage of topological indices is that they may be used directly as simple numerical descriptors in a comparison with physical, chemical, or biological parameters of molecules in quantitative structure-property relationships (QSPR) and in quantitative structure-activity relationships (QSAR). Therefore, one of the most interesting problems on topological indices is to study the extremal properties of topological indices and characterize the extremal structures. Another direction is to study the relation between topological indices and some other graph invariant, which would help people to know the indices better and find more internal information.

The quantification of chemical structures is a basic problem in structure-activity relationships (SAR) which is an important tool for developing safer and potent drugs. The procedure for quantification of chemical structures is by translation of chemical structures into characteristic numerical descriptors (topological indices) $[6,7,39,56]$. This procedure provides a correlation between quantitative biological activity and qualitative chemical structures. Based on this, Sardana and Madan [53] introduced a novel topological descriptor-adjacent eccentric distance sum index, which is defined as

$$
\xi^{s v}(G)=\sum_{v \in V(G)} \frac{\varepsilon(v) D(v)}{\operatorname{deg}(v)} .
$$

To verify that this index has a vast potential for SAR, Sardana and Madan $[53,54]$ selected different data sets to investigate some properties of adjacent eccentric distance sum index. In [53], to predict anti-HIV activity of 4,5,6,7-tetrahydro-imidazo- $[4,5,1-j k][1,4]$ benzodiazepin-2 $(1 \mathrm{H})$-one (TIBO) derivatives, the authors introduced this index which is computable. Moreover, the authors compared property of the adjacent eccentric distance sum index with that of the eccentric connectivity index and derived some excellent correlations between anti-HIV activity and both the topological descriptors. Their results show that the adjacent eccentric distance sum index has a potential for structure-activity/property studies. As we know, Wiener index is one of the most studied topological indices from the view of theory and applications, see for details [22, 23, 59]. In [54], the authors investigated the relationship of Wiener index and adjacent eccentric distance sum index with the antioxidant activity of nitroxides, as well as their hydroxylamine and amine precursors. In their investigation, authors selected 68 analogues whose antioxidant activity was reported. Firstly they computed the values of the Wiener 
index and the adjacent eccentric distance sum index for each analogues. Subsequently, each analogue was assigned a biological activity which was then compared with the reported antioxidant activity as $\mathrm{H}_{2} \mathrm{O}_{2}$ protection at a concentration of $100 \mu \mathrm{M}$. They supposed that compounds with an $\mathrm{H}_{2} \mathrm{O}_{2}$ protection of 2.5 or more were active and those with $\mathrm{H}_{2} \mathrm{O}_{2}$ protection of less than 2.5 or more were inactive. The overall degree of prediction was derived from the ratio of the total number of compounds predicted correctly to that of the total number of all compounds in both active and inactive ranges. Their results show that a total of 58 out of 68 compounds were classified correctly in both the active and inactive ranges with regard to Wiener index, but for adjacent eccentric distance sum index a total of 59 out of 68 compounds were classified correctly. The overall degree of prediction was found to 0.85 in case of Wiener index and 0.87 in case of adjacent eccentric distance sum index. These results encourage us to study the mathematical properties of this novel topological descriptor.

In this paper we derive some bounds for adjacent eccentric distance sum index in terms of some graph parameters, such as independence number, covering number, chromatic number, diameter and some graph topological indices.

\section{Methods}

Since adding edges would increase the degrees of some vertices and not increase $\varepsilon(v), D(v)$ for any vertex $v \in V(G)$, we have the following result.

Lemma 1 Let $G$ be a non-complete connected graph and e be an edge in $\bar{G}$ (the complement of $G)$. Then $\xi^{s v}(G)>\xi^{s v}(G+e)$.

The following result is immediate from Lemma 1.

Theorem 1 Let $G$ be a connected graph on $n$ vertices. Then

$$
\xi^{s v}(G) \geq n,
$$

with equality holding if and only if $G \cong K_{n}$.

If $G \neq K_{n}$, then

$$
\xi^{s v}(G) \geq n-2+\frac{4 n}{n-2},
$$

with equality holding if and only if $G \cong K_{n}-e$.

A graph is vertex-transitive if, for any two vertices $u$ and $v$, there is an automorphism $f$ of $G$ such that $f(u)=f(v)$. For a vertex-transitive graph $G$, it is regular and $r(G)=\varepsilon(w)$ for any $w \in V$ $(G)$. The Wiener index of a graph $G$, denoted by $W(G)$, is defined as the sum of the distances between all pairs of vertices in graph $G$, that is

$$
W(G)=\sum_{\{u, v\} \subseteq V(G)} d(u, v)=\frac{1}{2} \sum_{v \in V(G)} D(v) .
$$

Theorem 2 Let $G$ be a vertex-transitive graph on $n$ vertices with degree $\delta$. Then

$$
\xi^{s v}(G)=\frac{2 r(G)}{\delta} W(G) .
$$

The total eccentricity of the graph $G$, denoted by $\zeta(G)$, is defined as the sum of eccentricities of all vertices of graph $G$, i.e.,

$$
\zeta(G)=\sum_{v \in V(G)} \varepsilon(v) .
$$

It is sometimes interesting to consider it [17]. 
Theorem 3 Let $G$ be a connected graph on $n \geq 3$ vertices. Then

$$
\xi^{s v}(G) \geq \zeta(G)
$$

with equality holding if and only if $G \cong K_{n}$.

Proof. By fact that $D(v) \geq \operatorname{deg}(v)$ with equality holding if and only if $\varepsilon(v)=1$ and $\operatorname{deg}(v)=n$ -1 , i.e., $G \cong K_{n}$, we have

$$
\xi^{s v}(G) \geq \sum_{v \in V(G)} \frac{\varepsilon(v) \operatorname{deg}(v)}{\operatorname{deg}(v)}=\sum_{v \in V(G)} \varepsilon(v)=\zeta(G) .
$$

An independent set of $G$ is a subset $S$ of $V(G)$ such that no two vertices in $S$ are adjacent in $G$. The independence number of $G$, denoted by $\alpha(G)$, is the size of a maximum independent set of $G$. Let $\mathcal{G}(n, \alpha)$ be the set of all connected graphs with $n$ vertices and independence number $\alpha$. Let $G^{\diamond}$ denote the graph $K_{n-\alpha} \vee \overline{K_{\alpha}}$. Let $V\left(G^{\diamond}\right)=V_{1} \cup V_{2}$, where $V_{1}=\left\{v_{1}, v_{2}, \cdots, v_{\alpha}\right\}$ is the independent set $\overline{K_{\alpha}}$ of $G^{\diamond}$ and $V_{2}=\left\{v_{\alpha+1}, \cdots, v_{n}\right\}$ is the vertex set of $K_{n-\alpha}$. Let $e_{1}$ be an edge joining two vertices in $V_{1}$ and $V_{2}$. Let $e_{2}$ be an edge incident to two vertices in $V_{2}$.

Theorem 4 Let $G$ be a connected graph with $n$ vertices and independence number $\alpha$. Let $C=$

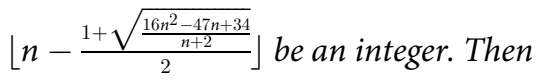

- $\xi^{s v}(G) \geq n-\alpha+\frac{2 \alpha(n+\alpha-2)}{n-\alpha}$, with equality holding if and only if $G \cong G^{\diamond}$;

- if $G \in \mathcal{G}_{n, \alpha} \backslash\left\{G^{\diamond}\right\}$, then

$$
\xi^{s v}(G) \geq\left\{\begin{array}{cl}
\frac{2(n+\alpha-2)(\alpha-1)}{n-\alpha}+\frac{2(n+\alpha-1)}{n-\alpha-1}+\frac{2 n}{n-2}+n-\alpha-1 & \text { if } 2 \leq \alpha \leq C, \\
\frac{2 \alpha(n+\alpha-2)}{n-\alpha}+\frac{4 n}{n-2}+n-\alpha-2 & \text { if } \alpha \geq C+1,
\end{array}\right.
$$

with equality holding if and only if $G \cong G^{\diamond}-e_{1}$ when $2 \leq \alpha \leq C ; G \cong G^{\diamond}-e_{2}$ when $\alpha \geq C+1$.

Proof. Assume that $G_{0}$ is a graph with the minimal adjacent eccentric distance sum index among all connected graphs on $n$ vertices with independence number $\alpha$. Let $S$ be a maximum independent set of $G_{0}$. Since adding edges would decrease the adjacent eccentric distance sum index, it follows that any vertex in $V\left(G_{0}\right)-S$ is adjacent to every vertex in $S$ and $G_{0}-S$ is complete. So $G_{0} \cong G^{\diamond}$. We have $\xi^{s v}\left(G^{\diamond}\right)=n-\alpha+\frac{2 \alpha(n+\alpha-2)}{n-\alpha}$.

By deleting an edge in $G^{\diamond}$, we get two graphs up to isomorphism: $G^{\diamond}-e_{1}, G^{\diamond}-e_{2}$. Since deleting an edge would increase the adjacent eccentric distance sum index, we only compare $\xi^{s v}\left(G^{\diamond}-e_{1}\right)$ with $\xi^{s v}\left(G^{\diamond}-e_{2}\right)$.

By some elementary calculations, we have

$$
\begin{aligned}
\xi^{s v}\left(G^{\diamond}-e_{1}\right) & =\frac{2(n+\alpha-2)(\alpha-1)}{n-\alpha}+\frac{2(n+\alpha-1)}{n-\alpha-1}+\frac{2 n}{n-2}+n-\alpha-1 \\
\xi^{s v}\left(G^{\diamond}-e_{2}\right) & =\frac{2 \alpha(n+\alpha-2)}{n-\alpha}+\frac{4 n}{n-2}+n-\alpha-2 .
\end{aligned}
$$

So we have

$$
\xi^{s v}\left(G^{\diamond}-e_{2}\right)-\xi^{s v}\left(G^{\diamond}-e_{1}\right)=\frac{n+2}{n-2}-\frac{4(n-1)}{(n-\alpha)(n-\alpha-1)} .
$$


Let $f(x)=\frac{n+2}{n-2}-\frac{4(n-1)}{(n-x)(n-x-1)}(2 \leq x \leq n-2)$ be a function. Taking derivation for $f(x)$, we have

$$
\frac{d f(x)}{d x}=-\left[\frac{4(n-1)}{(n-x)^{2}(n-x-1)}+\frac{4(n-1)}{(n-x)(n-x-1)^{2}}\right]<0 .
$$

So $f(x)$ is a strict decreasing function. It is easy to verify that $f(x) \geq 0$ if $x \leq\left\lfloor n-\frac{1+\sqrt{\frac{16 n^{2}-47 n+34}{n+2}}}{2}\right\rfloor$ and $f(x) \leq 0$ if $x \geq\left\lceil n-\frac{1+\sqrt{\frac{16 n^{2}-47 n+34}{n+2}}}{2}\right.$, which implies the result.

A covering of a graph $G$ is a subset $K$ of $V(G)$ such that every edge of $G$ has at least one endvertex in $K$. The covering number $\gamma$ of a graph $G$ is the number of vertices in any minimal covering. Since the complement of the maximum independent set is just the minimum covering, we have the following result.

Theorem 5 Let $G$ be a connected graph on $n$ vertices with covering number $\gamma$. Then

$$
\xi^{s v}(G) \geq \gamma+\frac{2(n-\gamma)(2 n-\gamma-2)}{\gamma},
$$

with equality holding if and only if $G \cong K_{\gamma} \vee \overline{K_{n-\gamma}}$.

A vertex cut of $G$ is a subset $V^{\prime}$ of $V$ such that $G-V^{\prime}$ is disconnected. A $\kappa$-vertex cut is a vertex cut of $\kappa$ elements. The vertex connectivity of a graph $G$, denoted by $\kappa(G)$, is the minimum $\kappa$ for which $G$ has a $\kappa$-vertex cut.

Theorem 6 Let $G$ be a connected graph with $n$ vertices and vertex connectivity $\kappa$. Then

$$
\xi^{s v}(G) \geq 2 n-\kappa+4(n-\kappa-1)\left(\frac{1}{\kappa}+\frac{1}{n-2}\right),
$$

with equality holding if and only if $G \cong K_{\kappa} \vee\left(K_{1} \cup K_{n-\kappa-1}\right)$.

Proof. Let $G_{0}$ be a graph with the minimal adjacent eccentric distance sum index among all connected graphs on $n$ vertices with vertex connectivity $\kappa$. Then there exists a vertex cut $S \subset V$ $\left(G_{0}\right)$ with $|S|=\kappa$ such that $G_{0}-S=G_{1} \cup G_{2} \cup \cdots \cup G_{t}$, where $G_{1}, G_{2}, \cdots, G_{t}(t \geq 2)$ are connected components of $G_{0}-S$. Since adding edges will decrease the adjacent eccentric distance sum index, the following three claims hold: (1) $t=2$; (2) the subgraphs $G_{1}, G_{2}$ and the induced subgraph $G_{0}[S]$ are complete; (3) any vertex of $G_{1} \cup G_{2}$ is adjacent to any vertex in $S$. Let $\left|G_{i}\right|=n_{i}$ for $i=1,2$ and $n_{1} \geq n_{2}$. Then $G_{0} \cong K_{\kappa} \vee\left(K_{n_{1}} \cup K_{n_{2}}\right)$ and $n_{1}+n_{2}=n-\kappa$.

By the definition of adjacent eccentric distance sum index, we have

$$
\xi^{s v}\left(G_{0}\right)=\kappa+2(n-\kappa)+4 n_{1} n_{2}\left(\frac{1}{n-n_{1}-1}+\frac{1}{n-n_{2}-1}\right) .
$$

Let $G_{0}^{\prime}=K_{\kappa} \vee\left(K_{n_{1}+1} \cup K_{n_{2}-1}\right)$. Then we consider the difference

$$
\begin{aligned}
\xi^{s v}\left(G_{0}\right)-\xi^{s v}\left(G_{0}^{\prime}\right)= & 4 n_{1} n_{2}\left(\frac{1}{n-n_{1}-1}+\frac{1}{n-n_{2}-1}\right) \\
& -4\left(n_{1}+1\right)\left(n_{2}-1\right)\left(\frac{1}{n-n_{1}-2}+\frac{1}{n-n_{2}}\right) \\
= & 4 \frac{\left(n_{1}-n_{2}+1\right)(n+\kappa-2)(\kappa-1)(n-1)}{\left(n-n_{1}-1\right)\left(n-n_{2}-2\right)\left(n-n_{2}\right)\left(n-n_{2}-1\right)}>0 .
\end{aligned}
$$

Therefore, $G \cong K_{\kappa} \vee\left(K_{1} \cup K_{n-\kappa-1}\right)$ has the minimal adjacent eccentric distance sum index among all connected graphs on $n$ vertices with vertex connectivity $\kappa$. 
Theorem 7 Let $G$ be a connected graph with $n$ vertices and minimum degree $\delta$. Then

$$
\xi^{s v}(G) \geq 2 n-\delta+4(n-\delta-1)\left(\frac{1}{\delta}+\frac{1}{n-2}\right),
$$

with equality holding if and only if $G \cong K_{\delta} \vee\left(K_{1} \cup K_{n-\delta-1}\right)$.

Proof. Let $G$ be a graph with the minimum adjacent eccentric distance sum index among connected graphs of order $n$ with minimum degree $\delta$. Assume that $G$ has vertex connectivity $\kappa$.

Let $f(x)=2 n-x+4(n-x-1)\left(\frac{1}{x}+\frac{1}{n-2}\right)$ be a function. It can be checked that $\frac{d f(x)}{d x}<0$ and therefore $f(x)$ is strictly decreasing. Hence $f(\kappa) \geq f(\delta)$ due to the fact that $\kappa \leq \delta$.

By Theorem 6, we have $\xi^{s v}(G) \geq f(\kappa) \geq f(\delta)$. If $\xi^{s v}(G)=f(\delta)$, then $\kappa=\delta$. This implies the result.

Similarly, we have

Theorem 8 Let $G$ be a connected graph on $n$ vertices with edge connectivity $\lambda$. Then

$$
\xi^{s v}(G) \geq 2 n-\lambda+4(n-\lambda-1)\left(\frac{1}{\lambda}+\frac{1}{n-2}\right),
$$

with equality holding if and only if $G \cong K_{\lambda} \vee\left(K_{1} \cup K_{n-\lambda-1}\right)$.

Theorem 9 Let $G$ be a connected graph on $n$ vertices with maximum degree $\triangle$. Then

$$
\xi^{s v}(G) \geq 2 n-\triangle+4(n-\triangle-1)\left(\frac{1}{\triangle}+\frac{1}{n-2}\right),
$$

with equality holding if and only if $G \cong K_{\triangle} \vee\left(K_{1} \cup K_{n-\triangle-1}\right)$.

The chromatic number of a graph $G$, denoted by $\chi(G)$, is the minimum number of colors such that $G$ can be colored with these colors and no two adjacent vertices have the same color.

Theorem 10 Let $G$ a connected graph on $n$ vertices with chromatic number $\chi$. Assume that $n$ $=\chi s+r$ with $1 \leq r<\chi$. Then

$$
\xi^{s v}(G) \geq \frac{2 s(\chi-r)(n+s-2)}{n-s}+\frac{2 r(s+1)(n+s-1)}{n-s-1},
$$

with equality holding if and only if $G \cong T_{n, \chi}$.

Proof. Let $G_{0}$ be a $\chi$-chromatic graph with minimal adjacent eccentric distance sum index. $G_{0}$ must be of the form $\overline{K_{1}} \vee \cdots \vee \overline{K_{\chi}}$. For any vertex $v \in V\left(\overline{K_{i}}\right), \varepsilon(v)=2, D(v)=n+n_{i}-2$ and $\operatorname{deg}(v)=n-n_{i}$. So we have

$$
\xi^{s v}\left(G_{0}\right)=\sum_{i=1}^{\chi} \frac{2 n_{i}\left(n+n_{i}-2\right)}{n-n_{i}}
$$

Assume that $n_{1} \leq n_{2} \cdots \leq n_{\chi}$. Let $G_{0}^{\prime}=\overline{K_{1}} \vee \cdots \vee \overline{K_{n_{i}+1}} \vee \cdots \vee \overline{K_{n_{j}-1}} \vee \cdots \vee \overline{K_{\chi}}$ if $2 \leq n_{i}<n_{j}$.

Considering the difference

$$
\begin{aligned}
\xi^{s v}\left(G_{0}\right)-\xi^{s v}\left(G_{0}^{\prime}\right)= & \frac{2 n_{i}\left(n+n_{i}-2\right)}{n-n_{i}}+\frac{2 n_{j}\left(n+n_{j}-2\right)}{n-n_{j}} \\
& -\frac{2\left(n_{i}+1\right)\left(n+n_{i}-1\right)}{n-n_{i}-1}-\frac{2\left(n_{j}-1\right)\left(n+n_{j}-3\right)}{n-n_{j}+1} \\
= & -\frac{4 n(n-1)\left(n_{j}-n_{i}-1\right)\left(n_{i}+n_{j}-2 n\right)}{\left(n-n_{i}\right)\left(n-n_{i}-1\right)\left(n-n_{j}\right)\left(n-n_{j}+1\right)} \geq 0 .
\end{aligned}
$$

Therefore, $T_{n, \chi}$ has the minimal adjacent eccentric distance sum index among all connected 
graphs on $n$ vertices with chromatic number $\chi$. By simple calculations, we have $\xi^{s v}\left(T_{n, \chi}\right)=\frac{2 s(\chi-r)(n+s-2)}{n-s}+\frac{2 r(s+1)(n+s-1)}{n-s-1}$.

A diametrical path in a graph is a path whose length equals the diameter of the graph. Let $P_{D+1}=v_{0} v_{1} \ldots v_{D}$ be a diametrical path in $G$. For the vertex $v_{0}$, the distance layer $L_{i}$ is the set of vertices $v \in G$ satisfying $d\left(v_{0}, v\right)=i$ for $i=0,1, \ldots, D$, i. e.

$$
L_{i}=\left\{v \mid d\left(v_{0}, v\right)=i\right\}, \quad i=0,1, \ldots, D
$$

In particular, $L_{\left\lceil\frac{D}{2}\right\rceil}$ is called the central layer.

The distance layer $L_{i}$ is called trivial if $\left|L_{i}\right|=1$. Obviously, $L_{0}$ is a trivial layer.

For $\overline{K_{2}}=\{u, v\},\left|l_{1}-l_{2}\right| \leq 1$ and $l_{1}+l_{2}=D-2$, the graph $G_{D}^{*}$ is obtained from $K_{n-D} \bigvee \overline{K_{2}}$ (the join of $K_{n-D}$ and $\overline{K_{2}}$ ) by identifying one end-vertex of each path of length $l_{1}$ and $l_{2}$ with $u$ and $v$, respectively. It is evident that its noncentral layers are trivial and any subgraph induced by the union of the central layer and its neighboring layer is complete.

For odd $D$, denote by $\mathcal{G}_{D}^{\diamond}$ the set of graphs obtained from $K_{k} \bigvee K_{n-D-1-k}$ by connecting an end-vertex of a path $P_{1}=P_{(D-1) / 2}$ with all vertices from $K_{k}$ and connecting an end-vertex of a path $P_{2}=P_{(D-1) / 2}$ with all vertices from $K_{n-D-1-k}$.

Theorem 11 Let $G$ be a connected graph of order $n$ with diameter D. Then

$$
\xi^{s v}(G) \geq \xi^{s v}\left(G_{D}^{*}\right)
$$

with equality holding if and only if $G \cong G_{D}^{*}$ for even $D$ and $G \in \mathcal{G}_{D}^{\diamond}$ for odd $D$.

Proof. Let $G_{0}$ be a graph with the minimal adjacent eccentric distance sum index among all connected graphs on $n$ vertices with diameter $D$. Assume that $P_{D+1}=v_{0} v_{1} \cdots v_{D}$ is a diametrical path in $G_{0}$. Since adding an edge decreases the adjacent eccentric distance sum index, the subgraphs induced by the union of two neighboring distance layers in $G_{0}$ are complete.

Suppose that $k \in\left\{1, \cdots,\left\lceil\frac{D+1}{2}\right\rceil-1\right\}$ is the smallest number such that $\left|V_{k}\right|>1$ in $G_{0}$. Let $v$ be a vertex in $V_{k}$, but different to $v_{k}$. We construct a new graph $G_{0}^{\prime}$ from $G_{0}$ by removing $v$ from $V_{k}$ to $V_{k+1}$ such that $G_{0}^{\prime}$ has the diameter $D$ and the subgraphs induced by the union of two neighboring distance layers in $G_{0}^{\prime}$ are complete. By the definition of adjacent eccentric distance sum index, we have

$$
\begin{aligned}
\xi^{s v}\left(G_{0}\right)= & \sum_{u \in V_{0} \cup \cdots \cup V_{k-1}} \frac{\varepsilon(u) D(u)}{\operatorname{deg}(u)}+\sum_{u \in V_{k}-\{v\}} \frac{\varepsilon(u) D(u)}{\operatorname{deg}(u)} \\
& +\sum_{u \in V_{k+1} \cup \cdots \cup V_{D}} \frac{\varepsilon(u) D(u)}{\operatorname{deg}(u)}+\frac{\varepsilon(v) D(v)}{\operatorname{deg}(v)} ; \\
\xi^{s v}\left(G_{0}^{\prime}\right)= & \sum_{u \in V_{0} \cup \cdots \cup V_{k-1}} \frac{\varepsilon(u)(D(u)+1)}{\operatorname{deg}(u)}+\sum_{u \in V_{k}-\{v\}} \frac{\varepsilon(u) D(u)}{\operatorname{deg}(u)} \\
& +\sum_{u \in V_{k+1} \cup \cdots \cup V_{D}} \frac{\varepsilon(u)(D(u)-1)}{\operatorname{deg}(u)}+\frac{(\varepsilon(v)-1)\left(D(v)-n+\left|V_{k}\right|+\left|V_{k+1}\right|+2 k\right)}{\operatorname{deg}(v)} .
\end{aligned}
$$


It follows that

$$
\begin{aligned}
\xi^{s v}\left(G_{0}\right)-\xi^{s v}\left(G_{0}^{\prime}\right)> & -\sum_{u \in V_{0} \cup \cdots \cup V_{k-1}} \frac{\varepsilon(u)}{\operatorname{deg}(u)}+\sum_{u \in V_{k+1} \cup \cdots \cup V_{D}} \frac{\varepsilon(u)}{\operatorname{deg}(u)} \\
= & \left(\sum_{u \in V_{D}} \frac{\varepsilon(u)}{\operatorname{deg}(u)}-\sum_{u \in V_{0}} \frac{\varepsilon(u)}{\operatorname{deg}(u)}\right)+\cdots \\
& +\left(\sum_{u \in V_{D-k+1}} \frac{\varepsilon(u)}{\operatorname{deg}(u)}-\sum_{u \in V_{k-1}} \frac{\varepsilon(u)}{\operatorname{deg}(u)}\right) \\
& +\sum_{u \in V_{k+1} \cup \cdots \cup V_{D-k}} \frac{\varepsilon(u)}{\operatorname{deg}(u)}
\end{aligned}
$$

Note that for any $1 \leq i \leq k-1$, we have $\varepsilon(u)=\varepsilon(w)$ for $u \in V_{i}, w \in V_{D-i}$ and

$$
\begin{aligned}
\sum_{w \in V_{D-i}} \frac{\varepsilon(w)}{\operatorname{deg}(w)}-\sum_{u \in V_{i}} \frac{\varepsilon(u)}{\operatorname{deg}(u)} & =(\operatorname{deg}(w)-1) \frac{\varepsilon(w)}{\operatorname{deg}(w)}-\frac{\varepsilon(u)}{2} \\
& =\left(\frac{\operatorname{deg}(w)-1}{\operatorname{deg}(w)}-\frac{1}{2}\right) \varepsilon(w) \geq 0
\end{aligned}
$$

For $i=0$, we have

$$
\sum_{w \in V_{D}} \frac{\varepsilon(w)}{\operatorname{deg}(w)}-\sum_{u \in V_{0}} \frac{\varepsilon(u)}{\operatorname{deg}(u)}=\varepsilon(w)-\varepsilon(u)=0 .
$$

It follows that

$$
\xi^{s v}\left(G_{0}\right)-\xi^{s v}\left(G_{0}^{\prime}\right)>\sum_{u \in V_{k+1} \cup \cdots \cup V_{D-k}} \frac{\varepsilon(u)}{\operatorname{deg}(u)}>0
$$

This contradict to the fact that $G_{0}$ has the minimal adjacent eccentric distance sum index in $\mathcal{G}_{n, D}$

Suppose that $k \in\left\{\left\lceil\frac{D+1}{2}\right\rceil+1, \cdots, D-1\right\}$ is the largest number such that $\left|V_{k}\right|>1$. Let $w$ be a vertex in $V_{k}$, but different to $v_{k}$. We construct a new graph $G_{0}^{\prime \prime}$ from $G_{0}$ by removing $w$ from $V_{k}$ to $V_{k-1}$ such that $G_{0}^{\prime \prime}$ has the diameter $D$ and the subgraphs induced by the union of two neighboring distance layers in $G_{0}^{\prime \prime}$ are complete. As above, we can get that $\xi^{s v}\left(G_{0}\right)>\xi^{s v}\left(G_{0}^{\prime \prime}\right)$, a contradiction.

Therefore, $G_{D}^{*}$ is the unique graph with minimal adjacent eccentric distance sum index in $\mathcal{G}_{n, D}$ for even $D$; for odd $D$, it can be seen that all graphs in $\mathcal{G}_{D}^{\diamond}$ have the same value $\xi^{s v}\left(G_{D}^{*}\right)$ and there are exactly $\left\lceil\frac{n-D}{2}\right\rceil$ extremal graphs with minimal adjacent eccentric distance sum index.

At last we shall calculate the value $\xi^{s v}\left(G_{D}^{*}\right)$. Assume that $P_{D+1}=v_{0} v_{1} \cdots v_{D}$ be a diametrical path in $G_{D}^{*}$. We divide the vertex set $V\left(G_{D}^{*}\right)$ into three parts: $V_{1}=\left\{v_{0}, v_{1}, \cdots, v_{\left\lceil\frac{D}{2}\right\rceil-1}\right\}, V_{2}=$ $\left\{v_{\left\lceil\frac{D}{2}\right\rceil+1}, \cdots, v_{D}\right\}$ and the central layer, i.e., $V_{3}=V_{\left\lceil\frac{D}{2}\right\rceil}$.

When $D$ is even, we have

- For $v_{i} \in V_{1}(i \geq 1)$, we have: $\varepsilon\left(v_{i}\right)=D-i$,

$$
\begin{aligned}
& D\left(v_{i}\right)=\frac{i(i+1)}{2}+\frac{(D-i)(D-i+1)}{2}+\left(\frac{D}{2}-i\right)(n-D-1), \operatorname{deg}\left(v_{i}\right)=2 ; \varepsilon\left(v_{0}\right)=D, \\
& D\left(v_{0}\right)=\frac{(D)(D+1)}{2}+\left(\frac{D}{2}\right)(n-D-1), \operatorname{deg}\left(v_{0}\right)=1
\end{aligned}
$$


- For $v_{i} \in V_{2}$, we have: $\varepsilon\left(v_{i}\right)=i$,

$D\left(v_{i}\right)=\frac{i(i+1)}{2}+\frac{(D-i)(D-i+1)}{2}+\left(i-\frac{D}{2}\right)(n-D-1), \operatorname{deg}\left(v_{i}\right)=2 ; \varepsilon\left(v_{D}\right)=D$,

$D\left(v_{D}\right)=\frac{D(D+1)}{2}+\left(D-\frac{D}{2}\right)(n-D-1), \operatorname{deg}\left(v_{D}\right)=1$

- For $v \in V_{3}$, we have: $\varepsilon(v)=\frac{D}{2}, D(v)=\frac{D(D+2)}{4}+n-D-1, \operatorname{deg}(v)=n-D+1$.

So we have

$$
\begin{aligned}
\xi^{d}\left(G_{D}^{*}\right)= & \frac{1}{2} \sum_{i=1}^{\frac{D}{2}-1}(D-i)\left[\frac{i(i+1)}{2}+\frac{(D-i)(D-i+1)}{2}+\left(\frac{D}{2}-i\right)(n-D)\right] \\
& +D\left[\frac{(D)(D+1)}{2}+\left(\frac{D}{2}\right)(n-D-1)\right] \\
& +\frac{1}{2} \sum_{j=\frac{D}{2}+1}^{D-1}\left[\frac{j(j+1)}{2}+\frac{(D-j)(D-j+1)}{2}+\left(j-\frac{D}{2}\right)(n-D)\right] \\
& +D\left[\frac{D(D+1)}{2}+\left(D-\frac{D}{2}\right)(n-D-1)\right] \\
& +\frac{1}{n-D+1} \frac{D(n-D)}{2}\left[\frac{D(D+2)}{4}+n-D-1\right] \\
= & -\frac{D\left(5 D^{4}+(15 n+43) D-\left(20 n^{2}-76 n+140\right) D^{2}\right)}{192(n-D+1)} \\
& +\frac{D\left(\left(144 n^{2}-84 n+28\right) D+112 n^{2}-80 n\right)}{192(n-D+1)} .
\end{aligned}
$$

Similarly, if $D$ is odd, for any $G \in \mathcal{G}_{D}^{\diamond}$, one has

$$
\begin{aligned}
\xi^{d}(G)= & \frac{1}{2} \sum_{i=1}^{\frac{D-1}{2}}(D-i)\left[\frac{i(i+1)}{2}+\frac{(D-i)(D-i+1)}{2}+\left(\frac{D+1}{2}-i\right)(n-D-1)\right] \\
& +D\left[\frac{(D)(D+1)}{2}+\left(\frac{D+1}{2}\right)(n-D-1)\right] \\
& +\frac{1}{2} \sum_{i=\frac{D+3}{2}}^{D-1}\left[\frac{i(i+1)}{2}+\frac{(D-i)(D-i+1)}{2}+i(n-D-1)-\frac{D+1}{2}(n-D-1)\right] \\
& +D\left[\frac{D(D+1)}{2}+\left(D-\frac{D+1}{2}\right)(n-D-1)\right] \\
& +\frac{1}{n-D+1}(n-D) \frac{D+1}{2}\left[\frac{(D+1)(D+3)}{8}+\frac{(D-1)(D+1)}{8}+n-D-1\right] \\
= & -\frac{5 D^{5}+(15 n+23) D^{4}-\left(20 n^{2}+96 n-66\right) D^{3}-\left(144 n^{2}-42 n+134\right) D^{2}}{192(n-D+1)} \\
& +\frac{\left(124 n^{2}-240 n+35\right) D+96 n^{2}-87 n-15}{192(n-D+1)} .
\end{aligned}
$$




\section{Discussion}

In this paper, we investigated the adjacent eccentric distance sum index and gave some lower bounds for this index in terms of some graph parameters such as independence number, covering number, vertex connectivity, chromatic number, diameter and some other topological indices. Moreover, the extremal graphs (attaining these bounds) are also characterized. Throughout this paper, the proof techniques used the structural properties of the graph parameters and the topological indices.

We only considered several graph parameters and topological indices in this work. To complete this knowledge, we shall consider relations between the adjacent eccentric distance sum index and other graph parameters and topological indices. In the future, we will continue to study extremal values of adjacent eccentric distance sum index for some special classes of graphs as well as for more general graphs. One direction of the future work is to consider the extremal values among some chemical or biology networks, such as benzenoid systems, fullerene graphs and polymeric networks.

\section{Acknowledgments}

The authors are grateful to anonymous referees from helpful comments to an earlier version of this manuscript.

\section{Author Contributions}

Conceived and designed the experiments: HQ SJC. Performed the experiments: HQ SJC. Contributed reagents/materials/analysis tools: HQ SJC. Wrote the paper: HQ SJC.

\section{References}

1. Al-Fozan T, Manuel P, Rajasingh I, Sundara Rajan R, Computing Szeged index of certain nanosheets using partition technique. MATCH Commun. Math. Comput. Chem. (2014) 72: 339-353.

2. Azari M, Iranmanesh A, Harary index of some Nano-structures. MATCH Commun. Math. Comput. Chem. (2014) 71: 373-382.

3. Bajaj S, Sambi SS, Gupta S, Madan AK, Model for prediction of anti-HIV activity of 2-pyridinone derivatives using novel topological descriptor, QSAR Comb. Sci. (2006) 25: 813-823.

4. Balaban AT, Chiriac A, Motoc I, Simon Z, Steric fit in QSAR, Lect. Notes Chem. (1980) 15: 22-27.

5. Balaban AT, Filip $P$, Computer programme for topological index (average distamce sum connectivity) $\mathrm{J}$. Math. Chem. (1984) 16: 163-190.

6. Balaban AT, Motoc I, Bonchev D, Mekennyan O, Topological indices for structure activity correlations, Top. Curr. Chem. (1983) 114:21-55.

7. Basak SC, Burtlsen S, Grunwold GD, Application of graph theoretical parameters in quantifying molecular similarity and structure activity relationships, J. Chem. Inf. Comput. Sci. (1994) 34:270-276. doi: 10.1021/ci00018a007

8. Bozkurt SB, Bozkurt D, Sharp upper bounds for energy and Randić energy, MATCH Commun. Math. Comput. Chem. (2013) 70: 669-680.

9. Bozkurt SB, Bozkurt D, On incidence energy. MATCH Commun. Math. Comput. Chem. (2014) 72: 215-225.

10. Bozkurt SB, Gutman I, Estimating the incidence energy. MATCH Commun. Math. Comput. Chem. (2013) 70: 143-156.

11. Cao S, Dehmer M, Shi Y, Extremality of degree-based graph entropies, Inform. Sciences (2014) 278 : 22-33. doi: 10.1016/j.ins.2014.03.133

12. Chen L, Liu J, Shi $Y$, Matching energy of unicyclic and bicyclic graphs with a given diameter, Complexity (2015) in press.

13. Chen L, Shi $Y$, The maximal matching energy of tricyclic graphs, MATCH Commun. Math. Comput. Chem. (2015) 73: 105-120. 
14. Chen Z, Dehmer M, Emmert-Streib F, Shi Y, Entropy bounds for dendrimers, Appl. Math. Comput. (2014) 242: 462-472. doi: 10.1016/j.amc.2014.05.105

15. Chen Z, Dehmer M, Shi Y, A note on distance-based graph entropies, Entropy (2014) 16: 5416-5427. doi: $10.3390 / \mathrm{e} 16105416$

16. Chen Z, Dehmer M, Shi Y, Yang Y, Sharp upper bounds for the Balaban index of bicyclic graphs, MATCH Commum. Math. Comput. Chem. (2015) in press.

17. Dankelmann $P$, Goddard W, Swart CS, The average eccentricity of a graph and its subgraphs, Util. Math. (2004) 65: 41-51.

18. Das KC, Gutman I, Cevik AS, Zhou B, On Laplacian energy. MATCH Commun. Math. Comput. Chem. (2013) 70: 689-696.

19. Das KC, Sorgun S, On Randić energy of graphs. MATCH Commun. Math. Comput. Chem. (2014) 72 : 227-238.

20. Dehmer M, Emmert-Streib F, Grabner M, A computational approach to construct a multivariate complete graph invariant. Information Sciences (2014) 260: 200-208. doi: 10.1016/j.ins.2013.11.008

21. Dehmer M, Li X, Shi Y, Connections between generalized graph entropies and graph energy, Complexity (2015) in press.

22. Dobrynin AA, Entringer R, Gutman I, Wiener index of trees: Theory and applications Acta Appl. Math. (2001) 66: 211-249. doi: 10.1023/A:1010767517079

23. Dobrynin A, Gutman I, Klavzar S, Zigert P, Wiener index of hexagonal systems, Acta Appl. Math. (2002) 72: 247-294.

24. Du W, Li X, Shi Y, Algorithms and extremal problem on Wiener polarity index, MATCH Commun. Math. Comput. Chem. (2009) 62: 235-244.

25. Dureja H, Gupta S, Madan AK, Predicting anti-HIV-1 activity of 6-arylbenzonitriles: Computational approach using superaugmented eccentric connectivity topochemical indices, J. Mol. Graph. Model. (2008) 26: 1020-1029. doi: 10.1016/j.jmgm.2007.08.008 PMID: 17919954

26. Fonseca CM, Stevanovic D, Further properties of the second Zagreb index. MATCH Commun. Math. Comput. Chem. (2014) 72: 655-668.

27. Ghorbani M, Faghani M, Ashrafi AR, Heidari Rad S, Graovac A, An upper bound for energy of matrices associated to an infinite class of fullerenes. MATCH Commun. Math. Comput. Chem. (2014) 71: 341354.

28. Gupta S, Singh M, Madan AK, Application of Graph Theory: Relationship of Eccentric Connectivity Index and Wiener's Index with Anti-inflammatory Activity, J. Math. Anal. Appl. (2002) 266: 259-268. doi: 10.1006/jmaa.2000.7243

29. Gupta S, Singh M, Madan AK, Eccentric distance sum: A novel graph invariant for predicting biological and physical properties, J. Math. Anal. Appl. (2002) 275: 386-401. doi: 10.1016/S0022-247X(02) 00373-6

30. Gupta S, Singh M, Madan AK, Connective eccentricity index: a novel topological descriptor for predicting biological activity, J. Mol. Graph. Model. (2000) 18: 18-25. PMID: 10935202

31. Gutman I, Li X, Shi Y, Zhang J, Hypoenergetic trees, MATCH Commun. Math. Comput. Chem. (2008) 60: 415-426.

32. Hosoya $\mathrm{H}$, Topological index; newly proposed quautity characterizing the topological nature of structure of isomers of saturated hydrocarbons, Bull. Chem. Soc. Jpn. (1971) 44: (1971) 2332-2337. doi: 10. 1246/bcsj.44.2332

33. Hosoya $\mathrm{H}$, Topological index as strong sorting device for coding chemical structure, J. Chem. Doc. (1972) 12: 181-183. doi: 10.1021/c160046a010

34. Hu Y, Li X, Shi Y, Xu T, Connected $(n, m)$-graphs with minimum and maximum zeroth-order general Randić index, Discrete Appl. Math. (2007) 155: 1044-1054.

35. Hu Y, Li X, Shi Y, Xu T, Gutman I, On molecular graphs with smallest and greatest zeroth-order general Randić index, MATCH Commun. Math. Comput. Chem. (2005) 54: 425-434.

36. Huo B, Li X, Shi Y, Complete solution to a problem on the maximal energy of unicyclic bipartite graphs, Linear Algebra Appl. (2011) 434: 1370-1377. doi: 10.1016/j.laa.2010.11.025

37. Huo B, Li X, Shi Y, Complete solution to a conjecture on the maximal energy of unicyclic graphs, European J. Combin. (2011) 32: 662-673.

38. Ilić A, Yu G, Feng L, On the eccentric distance sum of graphs, J. Math. Anal. Appl. (2011) 381:590600. doi: 10.1016/j.jmaa.2011.02.086

39. Kier LB, Hall LH, Molecular Connectivity in Sturcture Activity Analysis, Research Studies Press Ltd, Letchworth, (1986) pp. 1-257. 
40. Knor M, Luzar B, Skrekovski R, Gutman I, On Wiener index of common neighborhood graphs. MATCH Commun. Math. Comput. Chem. (2014) 72: 321-332.

41. Kumar V, Sardana S, Madan AK, Predicting anti-HIV activity of 2,3-diaryl-1,3 thiazolidin-4-ones: Computational approach using reformed eccentric connectivity index, J. Mol. Model. (2004) 10: 399407. doi: 10.1007/s00894-004-0215-8 PMID: 15597209

42. Kraus V, Dehmer M, Emmert-Streib F, Probabilistic inequalities for evaluating structural network measures. Information Sciences (2014) 288: 220-245. doi: 10.1016/j.ins.2014.07.018

43. Li H, Zhou Y, Su L, Graphs with extremal matching energies and prescribed parameters. MATCH Commun. Math. Comput. Chem. (2014) 72: 239-248.

44. Li R, Lower bounds for the Kirchhoff index. MATCH Commun. Math. Comput. Chem. (2013) 70: 163174.

45. Li X, Li Y, Shi Y, Gutman I, Note on the HOMO-LUMO index of graphs, MATCH Commun. Math. Comput. Chem. (2013) 70: 85-96.

46. Li X, Shi Y, A survey on the Randić index, MATCH Commun. Math. Comput. Chem. (2008) 59: 127156.

47. Li X, Shi Y, Gutman I, Graph Energy, (2012) Springer.

48. Li X, Shi Y, Wei M, Li J, On a conjecture about tricyclic graphs with maximal energy, MATCH Commun. Math. Comput. Chem. (2014) 72: 183-214.

49. Ma J, Shi Y, Yue J, The Wiener polarity index of graph products, Ars Combin. (2014) 116: 235-244.

50. Palacios JL, A resistive upper bound for the ABC index. MATCH Commun. Math. Comput. Chem. (2014) 72: 709-713.

51. Rada J, Cruz R, Gutman I, Benzenoid systems with extremal vertex-degree-based topological indices. MATCH Commun. Math. Comput. Chem. (2014) 72: 125-136.

52. Randić M, On characterization of molecular branching, J. Am. Chem. Soc. (1975) 97: 6609-6615. doi: 10.1021/ja00856a001

53. Sardana S, Madan AK, Predicting anti-HIV activity of TIBO derivatives: a computational approach using a novel topological descriptor, J. Mol. Model. (2002) 8: 258-265. PMID: 12324803

54. Sardana S, Madan AK, Relationship of Wiener's index and adjacent eccentric distance sum index with nitroxide free radicals and their precursors as modifiers against oxidative damage, J. Mol. Struct. (Theochem) (2003) 624: 53-59. doi: 10.1016/S0166-1280(02)00737-6

55. Sharma V, Goswami R, Madan AK, Eccentric connectivity index: A novel highly dis criminating topological descriptor for structure property and structure activity studies, J. Chem. Inf. Comput. Sci. (1997) 37: 273-282.

56. Trinajstic N, Chemical Graph Theory, Vols. I/II, CRC Press, Boca Raton, (1983).

57. Wiener $\mathrm{H}$, Correlation of heat of isomerization and difference in heat of vaporization of isomers among paraffin hydrocaibons, J. Amer. Chem. Soci. (1947) 69: 2636-2638. doi: 10.1021/ja01203a022

58. Wiener $\mathrm{H}$, Influence of interatomic forces on paraffin properties, J. Chem. Phys. (1947) 15: 766. doi: $10.1063 / 1.1746328$

59. Wiener H, Structural determination of the paraffin boiling points, J. Am. Chem. Soc. (1947) 69: 17-20. PMID: 20291038

60. Wiener $\mathrm{H}$, Vapour-pressure-temperature relations among the branched paraffin hydrocarbons, $\mathrm{J}$. Chem. Phys. (1948) 15: 425-430. doi: 10.1021/j150458a014

61. Xu K, Liu M, Das KC, Gutman I, Furtula B, A survey on graphs extremal with respect to distance-based topological indices. MATCH Commun. Math. Comput. Chem. (2014) 71: 461-508.

62. Yu G, Feng L, llić A, On the eccentric distance sum of trees and unicyclic graphs, J. Math. Anal. Appl. (2011) 375: 99-107. doi: 10.1016/j.jmaa.2010.08.054

63. Yu G, Feng L, On the connective eccentricity index of graphs, MATCH Commun. Math. Comput. Chem. (2013) 69:611-628.

64. Yu G, Qu H, Tang L, Feng L, On the connective eccentricity index of trees and unicyclic graphs with given diameter, J. Math. Anal. Appl. (2014) 420: 1776-1786. doi: 10.1016/j.jmaa.2014.06.050

65. Zheng W, Zou L, Lian X, Zhang H, Wang W, Zhao D, SQBC An efficient subgraph matching method over large and dense graphs. Information Sciences (2014) 261: 116-131. doi: 10.1016/j.ins.2013.10. 003 